\title{
Reaching Sub-Ångstrom Resolution with a Mid-Voltage TEM
}

\author{
Michael A. O’Keefe*, Crispin J.D. Hetherington ${ }^{\ddagger} \&$ E. Chris Nelson
}

National Center for Electron Microscopy, LBNL, 1 Cyclotron Road, Berkeley, CA 94720

${ }^{*}$ Current address: Materials Sciences Division, LBNL, 1 Cyclotron Road, Berkeley, CA 94720

${ }^{\ddagger}$ Current address: Department of Materials, Oxford University, Parks Rd, Oxford, OX1 3PH, UK

This document was prepared as an account of work sponsored by the United States Government. While this document is believed to contain correct information, neither the United States Government nor any agency thereof, nor The Regents of the University of California, nor any of their employees, makes any warranty, express or implied, or assumes any legal responsibility for the accuracy, completeness, or usefulness of any information, apparatus, product, or process disclosed, or represents that its use would not infringe privately owned rights. Reference herein to any specific commercial product, process, or service by its trade name, trademark, manufacturer, or otherwise, does not necessarily constitute or imply its endorsement, recommendation, or favoring by the United States Government or any agency thereof, or The Regents of the University of California. The views and opinions of authors expressed herein do not necessarily state or reflect those of the United States Government or any agency thereof, or The Regents of the University of California.

Ernest Orlando Lawrence Berkeley National Laboratory is an equal opportunity employer.

Ernest Orlando Lawrence Berkeley National Laboratory - LBNL-54892 


\title{
Reaching Sub-Ångstrom Resolution with a Mid-Voltage TEM
}

\author{
Michael A. O’Keefe*, Crispin J.D. Hetherington ${ }^{\ddagger} \&$ E. Chris Nelson
}

National Center for Electron Microscopy, LBNL, 1 Cyclotron Road, Berkeley, CA 94720

${ }^{*}$ Current address: Materials Sciences Division, LBNL, 1 Cyclotron Road, Berkeley, CA 94720

${ }^{\ddagger}$ Current address: Department of Materials, Oxford University, Parks Rd, Oxford, OX1 3PH, UK

The One-Ångstrom Microscope (OÅM) project was established at the NCEM to produce images at sub-Ångstrom resolution (O'Keefe, 1993). The project was implemented using a Philips CM300FEG/UT with hardware modifications designed to correct objective lens three-fold astigmatism and extend information transfer to 0.8 Ångstrom (O'Keefe et al., 2001a). A Gatan image filter (GIFTM) was used to bring the image magnification to more than three million times at the CCD camera to provide adequate real-space sampling.

Phase-contrast imaging in the HRTEM produces images with peaks at atom positions by extracting the spatial distribution of the relative phase from the electron wave. Usually, the electron wave is imaged by direct interference of diffracted beams at optimum focus (Scherzer, 1949). Instead, the OÅM uses the FEI Truelmage ${ }^{\mathrm{TM}}$ focal-series reconstruction software (Coene et al., 1996; Thust et al., 1996) to derive the relative electron phase from a series of images taken over a range of focus chosen to minimize the effects of partial spatial coherence (O'Keefe, 2001). The result of this focal-series reconstruction is to produce an experimental reconstruction of the electron wave at the specimen exit surface with a resolution that extends to the microscope information limit (O'Keefe, 1992). The spatial distribution of the relative phase of the reconstructed exit-surface wave (ESW) has peaks that correspond to the atom positions.

Careful design of the OÅM laboratory minimized the deleterious effects of vibration, electromagnetic noise and temperature fluctuations (Turner et al., 1997). In addition, it was found necessary to eliminate ground loops between the components of the microscope and its imaging filter. Specimen drift was minimized by setting the temperature of the cooling water exiting the objective lens coil so it matched the room temperature of $23^{\circ} \mathrm{C}$ and thus minimized temperature differences between the specimen, the specimen holder and the specimen stage. Low drift is essential to ensure sufficient overlap in the images of the focal series and provide enough common area for processing. Since the field of view on the CCD camera at 3.2 million times magnification is $150 \AA$ across, and acquisition of a 20 -member focal series of images takes two and one half minutes, any drift rate greater than $15 \AA /$ min would render the data set useless. To achieve maximum resolution, no talking or gross movements are allowed during the image series acquisition. In addition, we found that sudden atmospheric pressure changes have a significant effect -- opening or closing an external building door located 50 feet away is enough to ruin an acquisition series by introducing an abrupt change in defocus. An airlock system would alleviate this problem.

Tests using a silicon specimen tilted into [112] orientation showed that the OAM is capable of achieving resolutions down to $0.78 \AA$ (O'Keefe et al., $2001 \mathrm{~b}$ ). To produce this resolution, a series of 20 images was recorded, starting at an underfocus of $-3800 \AA$ and stepping towards overfocus in $11 \AA$ increments. The image drift was measured at $20.5 \AA$ over 148 seconds, or about $8.3 \AA$ $/ \mathrm{min}$. The reconstructed OÅM image shows white "blobs' representing pairs of silicon atoms arranged in "dumbbells" (a). A model of silicon in [112] orientation shows how the atoms are separated by $0.784 \AA$ in projection and demonstrates how they fit with the OÅM image (b). Resolution is the ability to determine how many objects (atoms in our case) a "blob" represents. Profiles of three of the "blobs" (arrowed in b) show that image peaks corresponding to the atom columns can just be distinguished (c), exceeding the Sparrow (1916) resolution criterion but not quite meeting that of Rayleigh (1874) which requires the dip between the peaks to be at $81 \%$ of the peak height. The "pixelation" visible in the image occurs because it is recorded with a sampling interval of $0.15 \AA$, so the horizontal period of $3.136 \AA$ (the silicon lattice parameter of $5.432 \AA$ divided by $\sqrt{ } 3$ ) is about 21 pixels, and the resolved atoms are separated by only 5.23 pixels. Using a higher magnification to provide finer sampling would provide insufficient area for reconstruction. 
The environment created by the OÅM laboratory has proven adequate to allow the OAM to reach its theoretical resolution of $0.8 \AA$ and to give the NCEM the capability to image columns of the lighter atoms, all the way down to lithium (Shao-Horn et al., 2003; O'Keefe \& Shao-Horn, 2004). Environments that are improved even more, such as those provided by the AML and Triebenberg laboratories, should prove more than adequate to allow the upcoming sub-Ångstrom TEMs and STEMs to reach their full potential.

\section{References:}

Coene, W.M.J., Thust, A., Op de Beeck, M. \& Van Dyck, D. (1996). Maximum-likelihood method for focus-variation image reconstruction in high resolution transmission electron microscopy. Ultramicroscopy 64, 109-135.

O'Keefe, M.A. (1992). 'Resolution' in high-resolution electron microscopy, Ultramicroscopy 47 282-297.

O'Keefe, M.A. (1993). Using coherent illumination to extend HRTEM resolution: why we need a FEG-TEM for HREM. LBL Symposium on Microstructures of Materials, edited by K. Krishnan (Berkeley: San Francisco Press), pp. 121-126.

O'Keefe, M.A. (2001). Alpha-null defocus: an optimum defocus condition with relevance for focalseries reconstruction. Microscopy and Microanalysis 7 (2001) supplement 2: 916-917.

O'Keefe, M.A., Hetherington, C.J.D., Wang, Y.C., Nelson, E.C., Turner, J.H., Kisielowski, C., Malm, J.-O., Mueller, R., Ringnalda, J., Pan, M., \& Thust, A. (2001a). Sub-Ångstrom highresolution transmission electron microscopy at 300keV. Ultramicroscopy 89, 4: 215-241.

O'Keefe, M.A., Nelson, E.C., Wang, Y.C. \& Thust, A. (2001b). Sub-Ångstrom resolution of atomistic structures below 0.8̊. Philosophical Magazine B 81, 11: 1861-1878.

O'Keefe, M.A. \& Yang Shao-Horn (2004). Sub-Ångstrom atomic-resolution imaging from heavy atoms to light atoms", Microscopy \& Microanalysis 10, 86-95.

Rayleigh, Lord (a.k.a. Strutt, J. W.). (1874). On the manufacture and theory of diffraction gratings. Philosophical Magazine 47, 310: 81-93.

Scherzer, O. (1949). The theoretical resolution limit of the electron microscope. J. Applied Physics, 20, 20-29.

Shao-Horn, Y., Croguennec, L., Delmas, C., Nelson, E.C. \& O'Keefe, M.A. (2003). Atomic resolution of lithium ions in $\mathrm{LiCoO}_{2}$ battery material. Nature Materials 2, 464-467.

Sparrow, C.M. (1916). On spectroscopic resolving power. Astrophys. Journal 44, 76-86.

Thust, A., Coene, W.M.J., Op de Beeck, M. \& Van Dyck, D. (1996). Focal-series reconstruction in HRTEM: simulation studies on non-periodic objects. Ultramicroscopy 64, 211-230.

Turner, J.H., O'Keefe, M.A. \& Mueller, R. (1997). Design and implementation of a site for a oneÅngstrom TEM. Microscopy and Microanalysis 3 (1997) supplement 2: 1177-1178.

\section{Acknowledgement:}

This work and the National Center for Electron Microscopy at LBNL are supported by the Director, Office of Science through the Office of Basic Energy Sciences, Material Sciences Division, of the U.S. Department of Energy, under contract No. DE-AC03-76SF00098. 

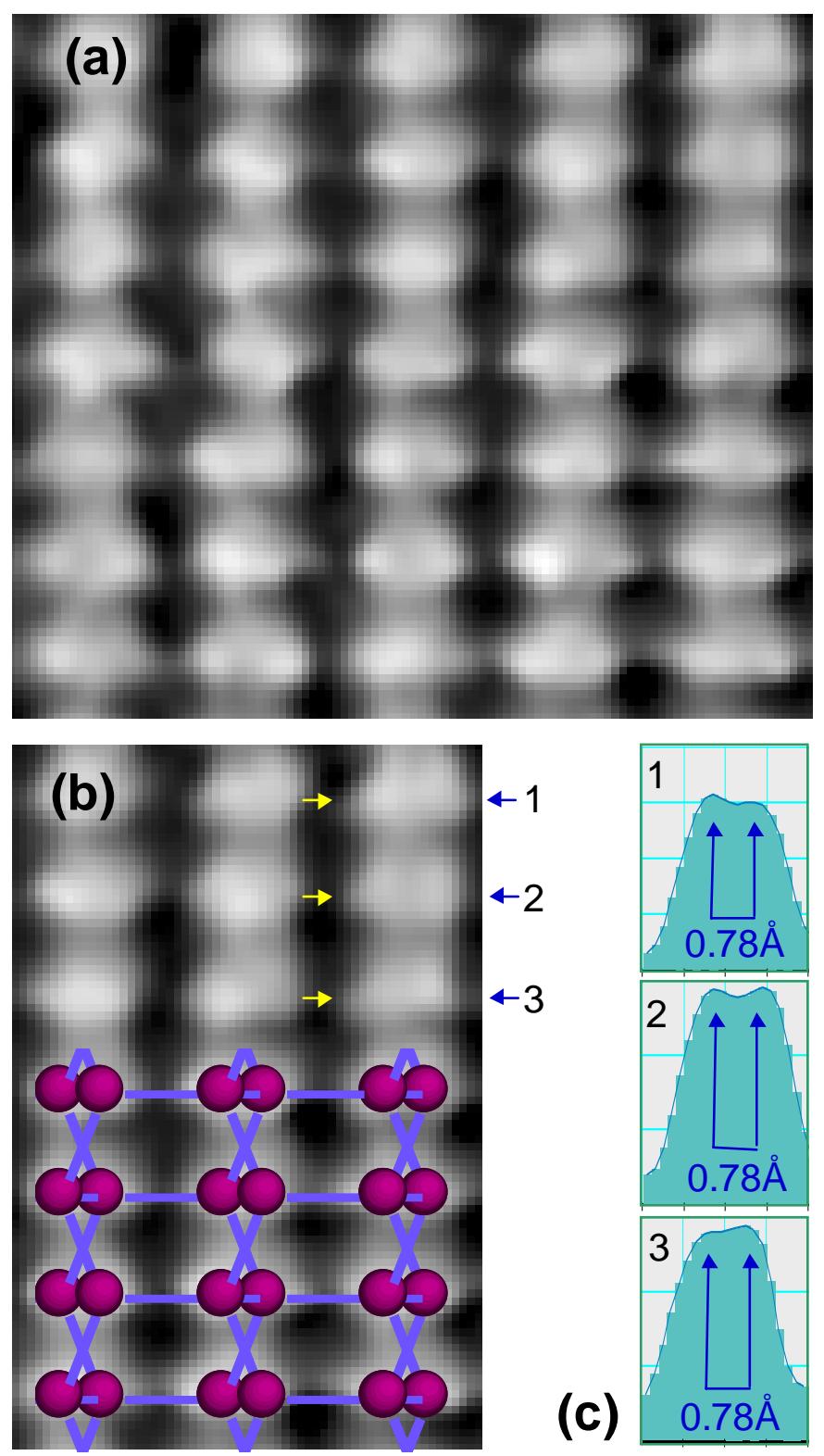

\section{Figure Caption:}

(a) OÅM image reconstructed from a focal series and displayed at 60 million times magnification. (b) With superimposed model of silicon in [112] orientation. (c) Profiles of three atom pairs (marked in b) with $0.784 \AA ̊$ scale bars. 